\title{
Three stage period analysis and complementary methods
}

\author{
L. Jetsu ${ }^{1,2}$ and J. Pelt ${ }^{3,4}$ \\ 1 Observatory, P.O. Box 14, University of Helsinki, FIN-00014, Finland \\ 2 NORDITA, Blegdamsvej 17, DK-2100 Copenhagen, Denmark \\ 3 Tartu Observatory, 61602 Tõravere, Estonia \\ 4 Institute of Theoretical Physics, P.O. Box 1029, Blindern, N-0315, Oslo, Norway
}

Received June 8, 1998; accepted August 6, 1999

\begin{abstract}
We generalize the multistage period search and analysis method for the case when the data error estimates (i.e. statistical weights) are available. Adjustment of model parameters allows us to detect complex wave forms in noisy data. Complementary statistical tests are also presented to estimate the significance of the detected periodicities, to verify the modelling reliability, and to separate real periodicities from spurious ones. The application of these methods is illustrated on different samples of real photometric data.
\end{abstract}

Key words: methods: numerical; data analysis; statistical

\section{Introduction}

The Lomb-Scargle periodogram (Lomb 1976; Scargle 1982; Press et al. 1988) is perhaps the most frequently applied period search and estimation method in astronomical time series analysis. The application of this method is somewhat restricted, because the underlying model for the data is a single harmonic. Several other methods have been reviewed by Cuypers (1986), while Schwartzenberg-Czerny (1996) and Foster (1996) represent some of the most recent developments. Pelt $(1980,1983,1992,1993)$ developed the general multistage period analysis for irregularly spaced data, where a crude period estimate is first obtained with an approximate pilot-search, and then refined at the subsequent stages. Since the above earlier versions of the multistage method were formulated only for data with equal measurement errors (unity weights), we present here a generalization for the case of unequal measurement errors. The following complementary statistical methods are also presented: the bootstrap for the model parameter error estimates, the evaluation of the modelling statistics

Send offprint requests to: jetsu@gstar.astro.helsinki.fi with the Kolmogorov-Smirnov test, and the identification of spurious periodicities from real ones with the phase residual regression. Thus the combination of weighted multistage period analysis and complementary statistical tests offers a flexible and powerful tool-set to search for periodicity in a large variety of astronomical time series. A formal presentation of these methods is illustrated on samples of photometric data of two variable stars (V 1794 Cyg and BL Her) and one nonvariable star (SAO50205). The complete data analysis of V 1794 Cyg is presented in Jetsu et al. (1999a, Paper I).

\section{Three stage period analysis (TSPA)}

A sample of $n$ data points $y\left(t_{i}\right)$ is fitted to a model $g(t, \bar{\beta})$ in our period search. The $Q$ free parameters are $\beta_{1}, \beta_{2}, \ldots$, $\beta_{Q}$. The most probable periodicity is searched for by determining the absolute minimum for the sum of the squared residuals

$\mathrm{RSS}=\sum_{i=1}^{n}\left[y\left(t_{\mathrm{i}}\right)-g\left(t_{i}, \beta_{1}, \ldots, \beta_{Q}\right)\right]^{2}$

Let us assume that the model can be expressed as a linear sum

$g\left(t, \beta_{1}, \ldots, \beta_{Q}\right)=\sum_{r=1}^{\mathrm{R}} \beta_{r} g_{r}\left(t, \beta_{R+1}, \ldots, \beta_{Q}\right)$

of separate model components $g_{r}\left(t, \beta_{R+1}, \ldots, \beta_{Q}\right)$, which depend nonlinearly on the periods (frequencies) or other parameters (e.g. decay rates, frequency shifts). In this case the RSS can be evaluated as a function of the nonlinear parameters only, because the linear parameters $\beta_{1}, \ldots, \beta_{R}$ can be estimated by ordinary regression methods for any fixed set of nonlinear parameters $\beta_{R+1}, \ldots, \beta_{Q}$. This gives the Least Squares Spectrum

$\operatorname{LSS}\left(\beta_{R+1}, \ldots, \beta_{Q}\right)=\sum_{i=1}^{n}\left[y\left(t_{i}\right)-g\left(t_{i}, \hat{\beta}_{1}, \ldots, \hat{\beta}_{R}, \beta_{R+1}, \ldots, \beta_{Q}\right)\right]^{2}$ 
where the linear parameter estimates $\hat{\beta}_{1}, \ldots, \hat{\beta}_{R}$ are also functions of the nonlinear parameters. The LSS minima reveal the most probable nonlinear parameter combinations. If the model is correctly specified, the global LSS minimum should give just the set of parameter estimates searched for. In this way the problem of model parameter estimation can be converted to the problem of solving the global minimum or minima of LSS. This approach has been applied to astronomical data already by Vaniček $(1969,1971)$ and Taylor \& Hamilton $(1972)$, or more recently by Martinez \& Koen (1994), Wilcox \& Wilcox (1995) and Bossi \& La Franceschina (1995). The LSS analysis is not trivial for semirandomly distributed observations, like the astronomical data that often contain periodic gaps. We formulate a flexible and computationally efficient approach to this problem by dividing the analysis into several stages, where the resolution of the spectra increases at each subsequent stage.

\subsection{Multistage search}

The first stage of the analysis, the Pilot Search, provides crude estimates for the nonlinear parameters. A standard Grid Search on a fine mesh centered at these crude estimates is performed during the second stage. Finally, traditional parameter refinement techniques are applied to obtain the highest possible precision (Refined Search). While the grid search and the refinement of the model parameters represent commonly used astronomical data processing techniques, the pilot search method is not so well known.

As a particular case of the general problem, we consider the LSS of the simple model

$g(f)=\hat{B}_{0}(f)+\sum_{r=1}^{R}\left[\hat{B}_{r}(f) \cos \left(r 2 \pi f t_{i}\right)+\hat{C}_{r}(f) \sin \left(r 2 \pi f t_{i}\right)\right]$, where the frequency $f$ is the only nonlinear free parameter. The linear free parameters for the mean $\left(\hat{B}_{0}\right)$ and the amplitudes $\left(\hat{B}_{r}, \hat{C}_{r}\right)$ are functions of $f$. The grid search over the full nonlinear parameter space tests a discrete frequency set $f_{l}=f_{\min }+(l-1) \Delta f$, where $l=1,2, \ldots, L$ and $f_{L}=f_{\max }$. The chosen $f_{\min }$ and $f_{\max }$ limits may represent the physically possible frequency range or some other interval of interest. The fixed frequency step $\Delta f$ must be chosen carefully. If the data cover an interval of $\Delta T=t_{n}-t_{1}$, it is reasonable constrain the maximum phase shift $\Delta \phi$ that can occur within $\Delta T$ when any tested $f_{l}$ changes by $\Delta f$. This gives $\Delta \phi=\Delta f \Delta T$. Sufficient values are $\Delta \phi \leq 0.1$, like the overfilling factor of $G=[\Delta \phi]^{-1} \geq 10$ in Sect. 3.2. Hence the corresponding number of tested frequencies becomes $L=\Delta T\left(f_{\max }-f_{\min }\right)[\Delta \phi]^{-1}$. If the tested periods are much shorter than $\Delta T$, then $L$ becomes large. Having fixed $\Delta \phi$, one can reduce $L$ by shortening $\Delta T$ or $f_{\max }-f_{\min }$. The following general scheme of a simple multistage search reduces $\Delta T$ on the first, and $f_{\max }-f_{\min }$ on the second stage, respectively.
1. Divide the data into $k$ separate parts with a length of $\Delta T_{k}$. Compute the LSS spectrum within each part using a frequency step $\Delta f_{\text {crude }}=\Delta \phi\left[\Delta T_{k}\right]^{-1}$, i.e. the number of tested frequencies is $L_{k}=\Delta T_{k}\left(f_{\max }-\right.$ $\left.f_{\min }\right)[\Delta \phi]^{-1}$. Use the average of all spectra for obtaining the crude periodicity estimates.

2. Compute the fine grid search spectra for all data within narrow frequency intervals centered at these crude estimates. Apply a frequency step of $\Delta f_{\text {fine }}=\Delta \phi[\Delta T]^{-1}$. A suitable width for the tested frequency interval is about $10\left\langle\Delta f_{\text {crude }}\right\rangle$, where $\left\langle\Delta f_{\text {crude }}\right\rangle$ is the mean frequency step that was used during the 1st stage. With this particular width, the number of tested frequencies is $L=10 \Delta T \Delta f_{\text {crude }}[\Delta \phi]^{-1}$.

3. Refine the precision of the frequency estimates with the standard Marquardt iteration (see e.g. Press et al. 1988).

The simplest multistage search techniques were first described by Evans (1961). Since then the computation of long spectra with powerful computers has become more popular than the application of complex multistage algorithms.

\subsection{Nonparametric dispersion estimation}

The average of the local LSS spectra gave the crude frequency estimates during the first stage of the simple multistage search described above. Pelt $(1983,1997)$ has introduced an alternative to compute these estimates. The idea is that if the data form a continuous periodic curve, the dispersion around this curve can be estimated without solving the curve itself. This dispersion is measured with the nonparametric test statistic

$\Theta(f)=\frac{\sum_{i=1, j=i+1}^{n-1, n} Z\left(t_{i}, t_{j}, f\right)\left[y\left(t_{i}\right)-y\left(t_{j}\right)\right]^{2}}{\sum_{i=1, j=i+1}^{n-1, n} Z\left(t_{i}, t_{j}, f\right),}$

where $Z\left(t_{i}, t_{j}, f\right) \geq 0$ deviates from zero only if

$t_{i}-t_{j} \approx k f^{-1}, k=0, \pm 1, \pm 2, \ldots$

The $\Theta(f)$ test statistic is a good approximation of the $\operatorname{LSS}(f)$ spectrum. A spectral window can be introduced like in the standard Fourier estimation. This is achieved with the more general formulation

$\Theta(f)=\frac{\sum_{i=1, j=i+1}^{n-1, n} Z\left(t_{i}, t_{j}, f\right) W\left(t_{i}, t_{j}\right)\left[y\left(t_{i}\right)-y\left(t_{j}\right)\right]^{2}}{\sum_{i=1, j=i+1}^{n-1, n} Z\left(t_{i}, t_{j}, f\right) W\left(t_{i}, t_{j}\right)}$

that allows additional smoothing with the data window

$W\left(t_{i}, t_{j}\right)= \begin{cases}1, & \left|t_{i}-t_{j}\right| \leq D_{\max } \\ 0, & \text { otherwise }\end{cases}$

The value of $D_{\max }=\Delta T$ gives the maximum resolution for the $\Theta(f)$ spectrum, because $W\left(t_{i}, t_{j}\right)=1$ for all pairs of observations. Adjusting this algorithm parameter to $D_{\max }<\Delta T$ enables a faster computation of a smoother spectrum. This reduces the number of tested frequencies 
during the first stage of the analysis, because a smaller $D_{\max }$ allows a longer step $\Delta f$ in the tested frequencies.

Although $\Theta(f)$ involves double summation for every tested frequency, this apparently complex test statistic can be computed effectively by rearranging these sums and using trigonometric approximations. Such computational techniques for different realizations of the algorithm can be found in (Pelt 1980, 1992). The application of this method speeds up the complete multistage analysis, because the $\Theta(f)$ spectrum for a full grid of tested frequencies can usually be computed as efficiently as the fast Fourier transform.

One particular version of a multistage analysis that can also utilize the additional information of the measurement errors is presented below.

\section{TSPA with weights}

This section contains the detailed formulation of a weighted three stage period analysis for the simple model of Sect. 2.1. The input data $y\left(t_{i}\right) \pm \sigma_{i}$ at $t_{1} \leq t_{2} \leq, \ldots, \leq t_{n}$ are $\bar{t}$ (time), $\bar{y}$ (observation) and $\bar{\sigma}$ (error), i.e. the weights are $\bar{w}$, where $w_{i}=\sigma_{i}^{-2}$. The $2 K+2$ free parameters $(\bar{\beta})$ for our $K$ :th order model

$g(\bar{\beta})=g(t, \bar{\beta})=M+\sum_{k=1}^{K} B_{k} \cos (k 2 \pi f t)+C_{k} \sin (k 2 \pi f t)$

are: the mean $(M)$, the amplitudes $\left(B_{k}, C_{k}\right)$ and the frequency $(f)$, i.e. $\bar{\beta}=\left[M, B_{1}, \ldots, B_{K}, C_{1}, \ldots, C_{K}, f\right]$. The definition for the phases is $\phi_{i}=\mathrm{FRAC}\left[f t_{i}\right]$, where FRAC removes the integer part of $f t_{i}$. The TSPA consists of three consecutive stages: the pilot- (PSch), the grid- (GSch) and the refined-search (RSch).

\subsection{Pilot Search (PSch)}

The order $K$ (Eq. 1) determines the PSch "model" $\tau$ (Eq. (2) below). The PSch searches for the best period "candidates" over a long interval between $P_{\min }\left(f_{\max }^{-1}\right)$ and $P_{\max }\left(f_{\min }^{-1}\right)$. Typical correlation lengths in time $\left(D_{\min }\right.$, $\left.D_{\max }\right)$ and phase $(\tau)$ are $2 P_{\max } \lesssim D_{\max } \lesssim 10 P_{\max }, D_{\min } \approx$ $0.9 P_{\min }$, and

$\tau=(4 K)^{-1}$.

Note that only $\tau$ is connected to our model (Eq. 1). The consequences of and the reasons for selecting adjustable correlation lengths $D_{\min }, D_{\max }$ and $\tau$ are discussed in the end of this section, as well the particular connection between $\tau$ and the modelling order $K$ (see also the beginning of Sect. 3.2).

The PSch uses four sets of $N=n(n-1) / 2$ pairs: $t_{i, j}$, $y_{i, j}, w_{i, j}$ and $\phi_{f, i, j}(i=1, \ldots, n-1$ and $j=i+1, \ldots, n)$. The first three, $t_{i, j}=\left|t_{i}-t_{j}\right|, y_{i, j}=\left[y\left(t_{i}\right)-y\left(t_{j}\right)\right]^{2}$ and $w_{i, j}=\left(w_{i} w_{j}\right)\left(w_{i}+w_{j}\right)^{-1}$, are independent of $f$, but the $\phi_{f, i, j}=\mathrm{FRAC}\left[f t_{i, j}\right]$ are not. The PSch periodogram determined at all integer multiples of $\Delta f_{\text {pilot }}=\left[10 D_{\max }\right]^{-1}$ between $f_{\min }$ and $f_{\max }$ is

$\Theta_{\text {pilot }}(f)=\frac{\sum_{i=1, j=i+1}^{n-1, n} Z\left(\phi_{f, i, j}\right) W\left(t_{i, j}\right) w_{i, j} y_{i, j}}{\sum_{i=1, j=i+1}^{n-1, n} Z\left(\phi_{f, i, j}\right) W\left(t_{i, j}\right) w_{i, j}}$,

where

$W\left(t_{i, j}\right)= \begin{cases}1, & D_{\min } \leq t_{i, j} \leq D_{\max } \\ 0, & \text { otherwise }\end{cases}$

$Z\left(\phi_{f, i, j}\right)= \begin{cases}1, & \phi_{f, i, j}<\tau \\ 1, & \phi_{f, i, j}>1-\tau \\ 0, & \text { otherwise. }\end{cases}$

The following algorithm is efficient for larger samples of data, because it eliminates the problem that the number of $t_{i, j}, y_{i, j}, w_{i, j}$ and $\phi_{f, i, j}$ increases with $\propto n^{2}$.

1. Because $W\left(t_{i, j}\right)$ does not depend on $f$ (Eq. 4), apply this function only once.

2. Divide $D_{\min } \leq t_{i, j} \leq D_{\max }$ into $J=\operatorname{INT}\left[\left(D_{\max }-\right.\right.$ $\left.\left.D_{\min }\right) \Delta^{-1}\right]+1$ bins $(J \ll N$ for larger samples $)$, where the bin width is $\Delta=10^{-1} P_{\min }$ and INT removes the decimal part of $\left(D_{\max }-D_{\min }\right) \Delta^{-1}$.

3. Denote the $n_{\mathrm{q}}$ values of $t_{i, j}, y_{i, j}$ and $w_{i, j}$ in the $q$ :th bin by $t_{k}^{\prime}, y_{k}^{\prime}$ and $w_{k}^{\prime}$. Derive $t_{q}^{\prime}=n_{q}^{-1} \Sigma_{k=1}^{n_{q}} t_{k}^{\prime}$, $y_{q}^{\prime}=\left[\Sigma_{k=1}^{n_{q}} w_{k}^{\prime}\right]^{-1} \Sigma_{k=1}^{n_{q}} w_{k}^{\prime} y_{k}^{\prime}$ and $w_{q}^{\prime}=\Sigma_{k=1}^{n_{q}} w_{k}^{\prime}$ for every bin.

4. Calculate the modified PSch periodogram

$\Theta_{\text {pilot }}^{\prime}(f)=\left[\sum_{q=0}^{J-1} Z\left(\phi_{f, q}^{\prime}\right) w_{q}^{\prime}\right]^{-1} \sum_{q=0}^{J-1} Z\left(\phi_{f, q}^{\prime}\right) w_{q}^{\prime} y_{q}^{\prime},(6)$

where $Z$ (Eq. 5) is applied to $\phi_{f, q}^{\prime}=\operatorname{FRAC}\left[f t_{q}^{\prime}\right]$.

This algorithm divides the data into $J$ bins with respect to the time differences $t_{i, j}$ between $D_{\min }$ and $D_{\max }$. The original $t_{i, j}, y_{i, j}$ and $w_{i, j}$ data are replaced by the $J$ averages $t_{q}^{\prime}, y_{q}^{\prime}$ and $w_{q}^{\prime}$ within these bins. The algorithm is efficient, because the $Z$ function in Eq. (6) has to applied only to $J(\ll N)$ values at each tested $f$.

The $y_{i, j}$ differences closer than $\tau$ in $\phi_{f, i, j}$ are smaller for a good $f$ candidate, i.e. the data form a continuous curve. Such $f$ minimize the PSch periodogram (Eqs. 3 or 6 ), the case being opposite for poor $f$ candidates. Because $W\left(t_{i, j}\right)$ (Eq. 4) excludes $y_{i, j}$ too far in $t_{i, j}$, phase shifts during time intervals longer than $D_{\max }$ do not influence the periodogram. The combination of $D_{\max }=\Delta T$ and $D_{\text {min }}=0$ would include all data (Eq. 4), but $D_{\min }$ is applied, because $y_{i, j}$ do not contain significant information when $t_{i, j}$ goes below $P_{\min }$. Adjusting $D_{\min }$ and $D_{\max }$ determines the number of $y_{i, j}$ selected with $W\left(t_{i, j}\right)$. The function $Z\left(\phi_{f, i, j}\right)$ selects only $y_{\mathrm{i}, \mathrm{j}}$ closer than $\tau$ in $\phi_{f, i, j}$ (Eq. 5). For example, $\tau=0.25$ determines a sinusoidal model (Eqs. 1 and 2). The $y_{i, j}$ on a sinusoid correlate within $\phi_{f, i, j}= \pm 0.25$, or those on a double wave $(K=2)$ within $\phi_{f, i, j}= \pm 0.125$. Reduction of $\tau$ enables detection of more complex $\bar{y}$ variation, but reduces the number of $y_{i, j}$ selected with $Z\left(\phi_{f, i, j}\right)$, i.e. requires more data. 


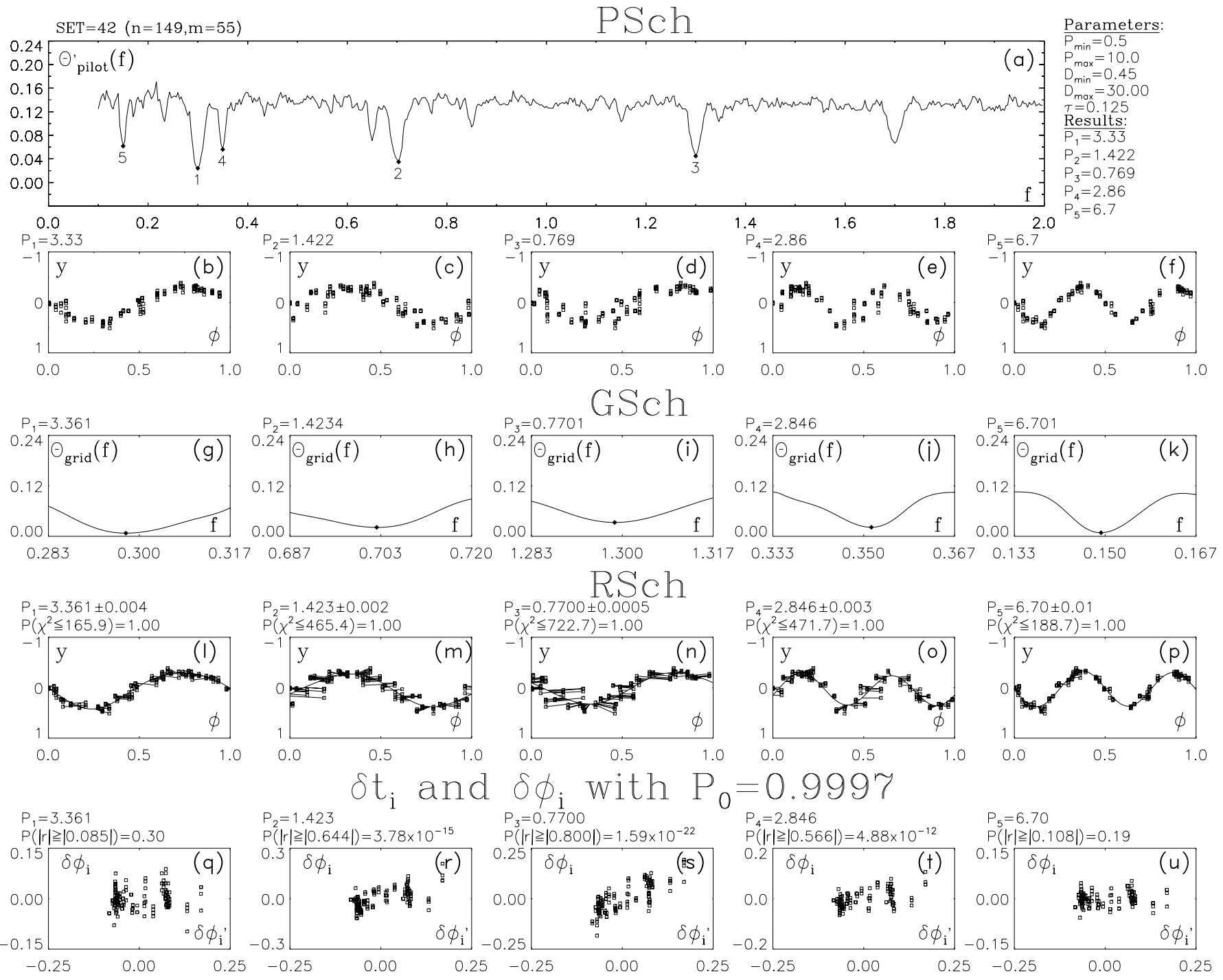

Fig. 1. The second order TSPA for SET $=42(n=149)$ : a) $\Theta_{\text {pilot }}^{\prime}(f)$ (Eq. 6) is the PSch periodogram between $P_{\min }=0.5$ and $P_{\max }=10$ with correlation lengths $D_{\min }=0.45, D_{\max }=30$ and $\tau=0.125$. The number of independent frequencies is $m=55$ (Eq. 9). The diamonds on $\Theta_{\text {pilot }}(f)$ mark the five best periods $P_{1}, \ldots, P_{5}$. b-f) The $y_{i}\left(\phi_{i}\right)$ with these $P_{1}, \ldots, P_{5}$. g-k) The diamonds on $\Theta_{\text {grid }}(f)$ (Eq. 7: $K=2$ ) indicate the more accurate $P_{1}, \ldots, P_{5}$ obtained with the GSch. l-p) RSch determines the final $P_{1}, \ldots, P_{5}$, and their $P\left(\chi_{0}^{2}\right)$ (Eq. 10). The continuous lines connect each $\left(\phi_{i}, y_{i}\right)$ to the closest point of the model $(\phi, g(\phi))$. q-u) The $\delta \phi_{i}^{\prime}$ versus $\delta \phi_{i}$ (see end of Sect. 5) of each $P_{1}, \ldots, P_{5}$ for $P_{0}=0.9997$ given by $\gamma_{\mathrm{n}}$ (Eq. 15). The critical levels for the linear correlations between $\delta \phi_{i}^{\prime}$ and $\delta \phi_{i}$ are $P\left(\left|r_{0}\right|\right)$

\subsection{Grid Search (GSch)}

The PSch over a long $f$ interval usually detects numerous frequency "candidates" $f^{\prime}$. More accurate values for at least five best $f^{\prime}$ are determined with the GSch. The TSPA applications for real data may sometimes require testing of a much larger number of $f^{\prime}$ detected with the PSch. Here the limit of testing only the five best $f^{\prime}$ was chosen, because it is convenient for the graphical representation of the results, like in Figs. 1, 3, 5 and 7. The PSch "model" $\tau$ can not fully constrain the modelling order $K$ (Eqs. 1 and 2), because $y_{i, j}$ closer than $\tau$ in $\phi_{f, i, j}$ may correlate in several different models. For example, the PSch could detect a box function or a sinusoid with $\tau=0.25$. The model is fixed in the GSch, e.g. if $\tau=0.25$, GSch proceeds with a $K=1$ model (Eq. 1). If PSch detects $f^{\prime}$, then GSch tests all integer multiples of $\Delta f_{\text {grid }}=[G]^{-1} f_{0}$ between $f^{\prime}-5 \Delta f_{\text {pilot }}$ and $f^{\prime}+5 \Delta f_{\text {pilot }}$, where $G \geq 10$ is called the "overfilling factor" and $f_{0}=(\Delta T)^{-1}$. The discrete tested $f$ set within this narrow interval is denser than in the PSch (i.e. $\Delta f_{\text {grid }} \leq \Delta f_{\text {pilot }}$ ). Standard linear least squares fits to $\bar{y}$ with $\bar{w}$ for every tested $f$ in Eq. (1) (i.e. $f$ is not a free parameter) determine the GSch periodogram

$\Theta_{\operatorname{grid}}(f)=2\left[\sum_{i=1}^{n} w_{i}\right]^{-1} \sum_{i=1}^{n} w_{i}\left[y\left(t_{i}\right)-g\left(t_{i}, \bar{\beta}_{f}\right)\right]^{2}$ 

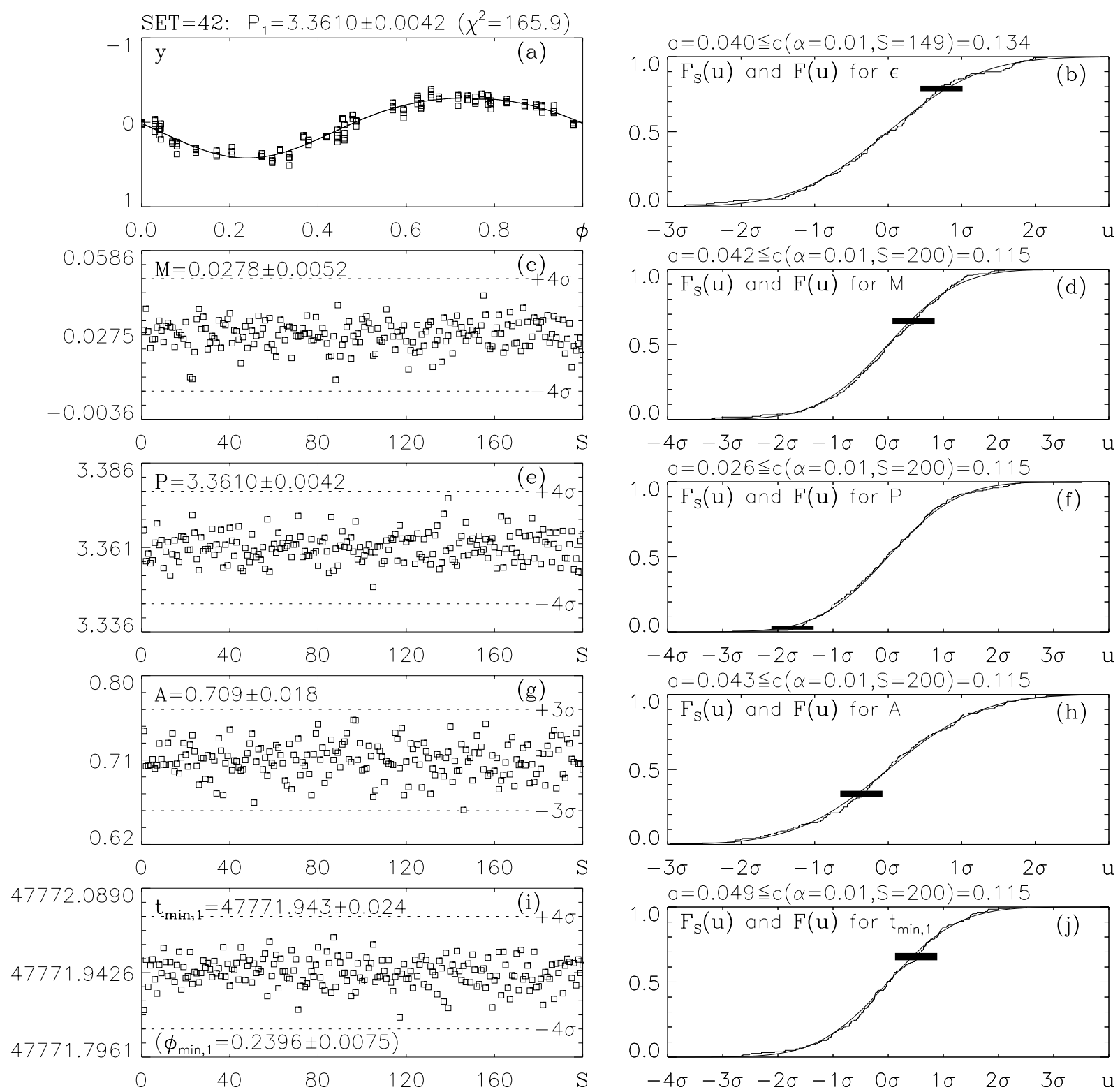

Fig. 2. The $S=200$ RSch bootstrap of $\mathrm{SET}=42$ : a) The model $(\bar{g})$ with $P_{1}=3.3610 \pm 0.0042$ already shown in Fig. 1l. b) The $F_{\mathrm{S}}(u)$ and $F(u)$ (Eqs. 12 and 13) for the $S=149(=n)$ residuals $\bar{\epsilon}$ confirm a Gaussian distribution (i.e. $H_{\mathrm{G}}$ is not rejected with $\alpha=0.01$ in Eq. 14). A dark rectangle indicates the location and height of the Kolmogorov-Smirnov test statistic $\left(a=\max \left[\left|F_{\mathrm{S}}(u)-F(u)\right|\right]\right)$. c and d) The $S=200$ bootstrap $M$ estimates (open squares) also follow a Gaussian distribution. e-j) The same as in c and d) for the $P, A$ and $t_{\min , 1}$ estimates

where $\bar{\beta}_{f}=\left[M, B_{1}, \ldots, B_{K}, C_{1}, \ldots, C_{K}\right]$ minimizes the residuals $\epsilon_{i}=y\left(t_{i}\right)-g\left(t_{i}, \bar{\beta}_{f}\right)$ (note that $\Theta_{\text {grid }} \neq \chi^{2}$ ), and the factor 2 adjusts $\Theta_{\text {grid }}$ to the quantitative level of $\Theta_{\text {pilot }}$. Thus the PSch periodogram with $\tau=(4 K)^{-1}$ represents an approximation of the GSch periodogram with $K$.

\subsection{Refined Search (RSch)}

The model (Eq. 1) is nonlinear when $f$ is a free parameter. The RSch performs a standard Marquardt iteration (e.g. Press et al. 1988) to compute $\bar{\beta}$. But the result of this iter- ative refinement depends on the trial solution $\left(\bar{\beta}_{0}\right)$. Large discrete $f$ sets are tested with PSch and GSch only to provide a reliable $\bar{\beta}_{0}$ for RSch. Combining the $f^{\prime}$ detected in the GSch to $\bar{\beta}_{f^{\prime}}$ (Eq. 7) gives $\bar{\beta}_{0}=\left[\bar{\beta}_{f^{\prime}}, f^{\prime}\right]$. While the PSch and GSch test discrete $f$ sets, the RSch is continuous in $f$, because it utilizes the analytical properties of

$\chi^{2}\left(\bar{\beta}_{\min }\right)=\sum_{i=1}^{n} w_{i}\left[y\left(t_{i}\right)-g\left(t_{i}, \bar{\beta}_{\min }\right)\right]^{2}$,

where $\bar{\beta}_{\text {min }}$ minimizes the $\chi^{2}$. This $\chi^{2}$ enables significance estimates. The number of independent frequencies $(m)$ determines how many independent $\chi^{2}$-tests are done with 


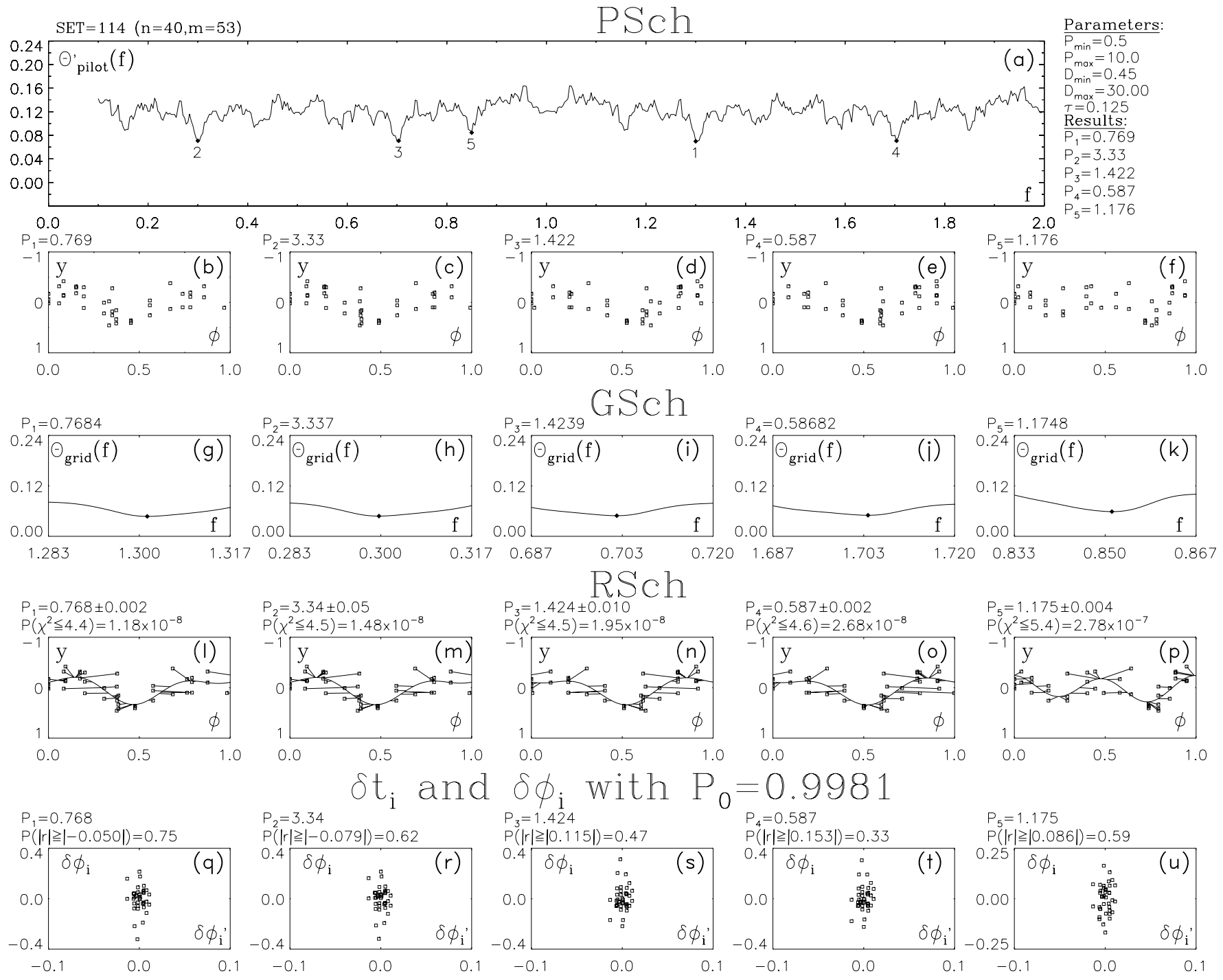

Fig. 3. Same as in Fig. 1 for $\mathrm{SET}=114$

the model of Eq. (1). Since the TSPA is performed between $f_{\min }\left(P_{\max }^{-1}\right)$ and $f_{\max }\left(P_{\min }^{-1}\right)$, a logical definition is $m=\operatorname{INT}\left[\left(f_{\max }-f_{\min }\right) f_{0}^{-1}\right]$,

where $f_{0}=\Delta T^{-1}$. This definition is equivalent to that in Jetsu \& Pelt (1996, Eq. 13), who presented an empirical approach to verify Eq. (9) (see also Buccheri \& De Jager 1989). To understand the connection between $f_{0}$ and $m$, consider an arbitrary test statistic $z(f)$ that depends on $\phi_{i}=\mathrm{FRAC}\left[f t_{i}\right]$. Because $\Delta T\left(f \pm f_{0}\right)-\Delta T f= \pm 1$ for any $f$, the correlation between $z(f)$ values vanishes within $f_{0}$ when the $\phi_{i}$ order is totally rearranged. An overfilling factor, e.g. $G \geq 10$ in GSch, enables more accurate period determination, but does not deteriorate the statistics, i.e. testing many frequencies within $\pm f_{0} / 2$ amounts to testing only one independent frequency. Hence the TSPA performs $m$ independent $\chi^{2}$-tests over the frequency interval $\left[f_{\min }, f_{\max }\right]$. Our "null hypothesis" is:

$H_{0}$ : The data are pure noise.
Under $H_{0}$, the probability (i.e. the critical level) for reaching a particular, or an even smaller, $\chi_{0}^{2}$ value, is

$P\left(\chi_{0}^{2}\right)=P\left(\chi^{2} \leq \chi_{0}^{2}, \nu, m\right)=1-\left[1-P\left(\chi^{2} \leq \chi_{0}^{2}\right)\right]^{\mathrm{m}}$,

where $\nu=n-(2 K+2)$ is the degree of freedom for $\chi^{2}$. The validity of $\chi^{2}$ statistics is verified in the next section by testing the hypothesis that the model residuals and parameters have a Gaussian distribution.

\section{Bootstrap}

Theoretical (e.g. $\chi^{2}$ ) and numerical (e.g. Monte Carlo) $\bar{\beta}_{\text {min }}$ error estimates for nonlinear models are discussed, e.g. by Press et al. (1988). We estimate the $\bar{\beta}_{\text {min }}$ errors with the bootstrap for the regression coefficients (Efron \& Tibshirani 1986). This bootstrap also allows estimates of "other" model parameters (and their errors) that would 

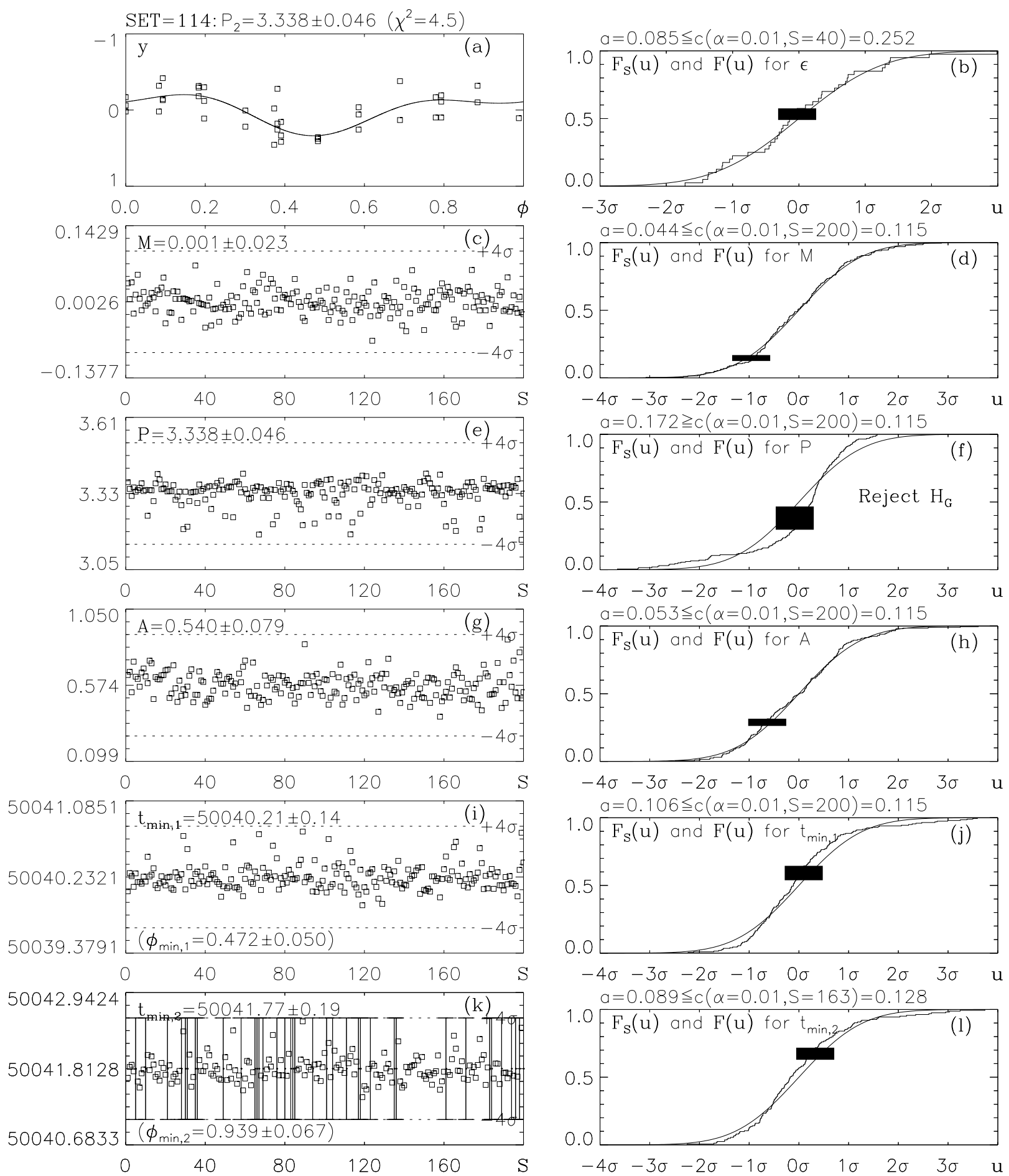

Fig. 4. Same as in Fig. 2 for SET=114. Note: e and f) $H_{\mathrm{G}}$ is rejected for $P$ (Eq. 14). k) Only $S=163$ estimates are obtained for $t_{\mathrm{min}, 2}$. The bootstrap samples with no $t_{\mathrm{min}, 2}$ are marked with vertical lines

be difficult to solve analytically for $K \geq 2$, e.g. the total amplitude $(A)$ and the epochs of the minima $\left(t_{\min , 1}\right.$, $\left.t_{\min , 2}, \ldots, t_{\min , \mathrm{K}}\right)$. The six stages of our bootstrap are:

1. Minimizing $\chi^{2}$ (Eq. 8) for $\bar{y}$ with $\bar{w}$ gives $\epsilon_{i}=y\left(t_{i}\right)-g\left(t_{i}, \bar{\beta}_{\min }\right)=y_{i}-g_{i}$,
2. A random sample $\bar{\epsilon}^{*}$ is selected from $\bar{\epsilon}$. The number of the same $\epsilon_{i}$ entering into $\bar{\epsilon}^{*}$ may vary between 0 and $n$ in this random sample with replacement. The $\bar{\epsilon}^{*}$ determine a unique random sample $\bar{w}^{*}$, where the connection of $w_{i}$ being the weight of $\epsilon_{i}$ is preserved.

3. A random sample $\bar{y}^{*}=\bar{g}+\bar{\epsilon}^{*}$ is obtained.

i.e. the "empirical distribution of residuals". 


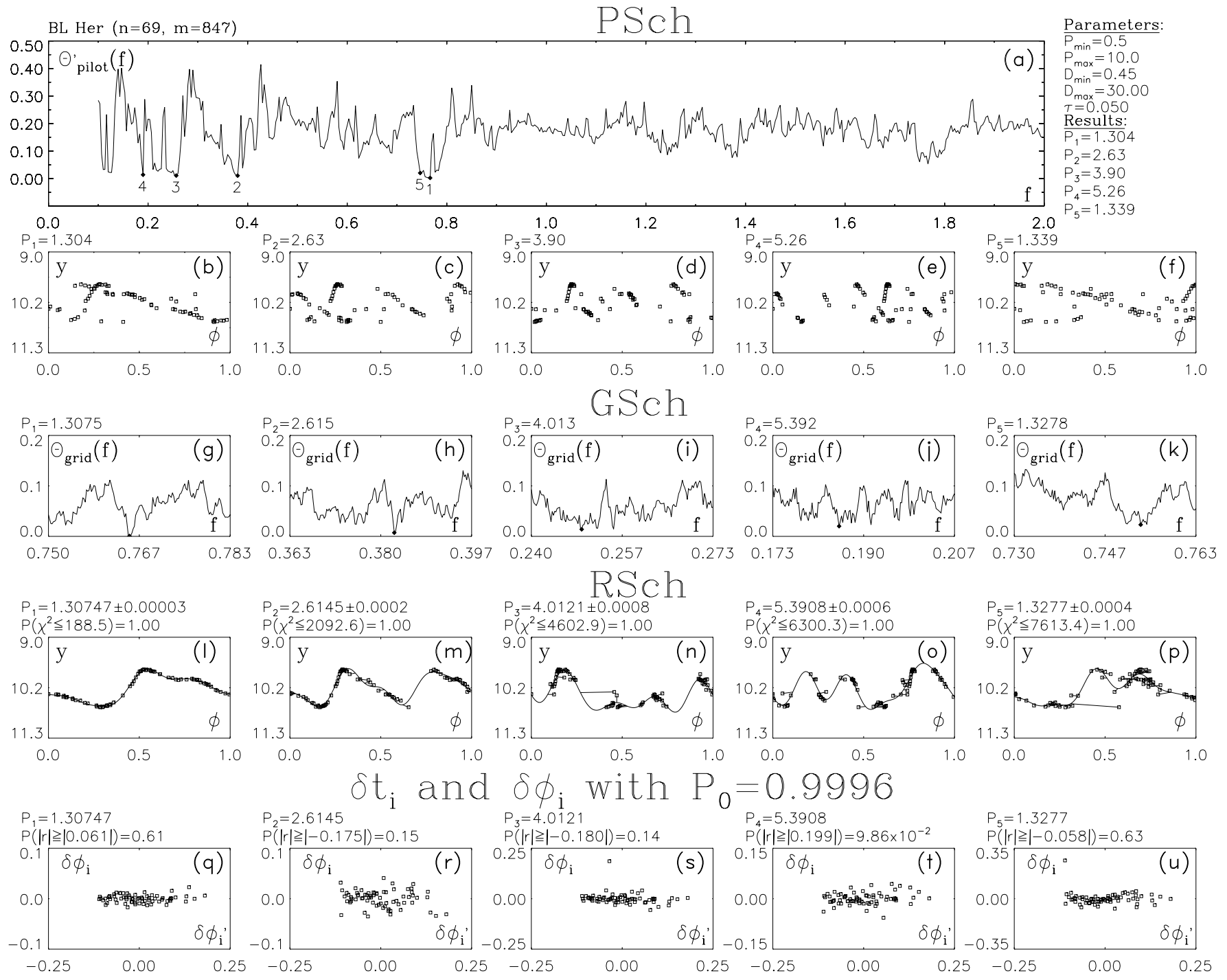

Fig. 5. The fifth order TSPA $(K=5)$ for the $V$ magnitudes of the cepheid variable BL Her, otherwise as in Fig. 1

4. Minimizing $\chi^{2}$ (Eq. 8) for $\bar{y}^{*}$ with $\bar{w}^{*}$ gives one estimate for $\bar{\beta}^{\prime}{ }_{\min }$, as well as for the "other" parameters of higher order models $(K \geq 2)$. For example, measuring the difference between the minimum and maximum of $\bar{g}\left(\bar{\beta}^{\prime}{ }_{\text {min }}\right)$ gives a numerical $A$ estimate.

5. The bootstrap returns to the 2 nd stage, until $S$ estimates of $\bar{\beta}^{\prime}{ }_{\min }$ and "other" parameters have been obtained.

6. The expectation value and variance for any $\bar{\beta}_{\text {min }}$ component are the mean and variance of its $S$ estimates in $\bar{\beta}_{\text {min }}^{\prime}$. The same applies to the $S$ estimates of "other" model parameters.

Note that the $\bar{y}, \bar{w}, \bar{\epsilon}$ and $\bar{g}$ remain unchanged, while $\bar{y}^{*}$, $\bar{w}^{*}$ and $\bar{\epsilon}^{*}$ are changing, and $\bar{\epsilon}^{*}$ determines both $\bar{y}^{*}$ and $\bar{w}^{*}$.

We introduce a few notations useful in testing the "Gaussian hypothesis"
$H_{\mathrm{G}}$ : An arbitrary $\bar{x}$ with $S$ components represents a random sample drawn from a Gaussian distribution.

These $\bar{x}$ (e.g. $\bar{\epsilon}, \bar{M}, \bar{A}, \bar{P}$ or $\bar{t}_{\mathrm{min}, 1}$ ) are first arranged into an ascending (i.e. rank) order $x_{1} \leq x_{2} \leq \ldots \leq x_{\mathrm{S}}$ and then transformed to $u_{i}=\left(x_{i}-m_{x}\right) / s_{x}$, where $m_{x}$ and $s_{x}$ are the mean and standard deviation of $\bar{x}$. The cumulative distribution function is

$F_{\mathrm{S}}(u)=\left\{\begin{array}{l}0, u<u_{1} \\ i S^{-1}, u_{i} \leq u<u_{i+1} \\ 1, u>u_{\mathrm{S}}\end{array}\right.$

and the cumulative Gaussian distribution function is

$F(u)=(2 \pi)^{-1 / 2} \int_{-\infty}^{\mathrm{u}} \mathrm{e}^{-\mathrm{z}^{2} / 2} \mathrm{~d} z$.

The preassigned significance level $\alpha=0.01$ for rejecting $H_{\mathrm{G}}$ determines an upper limit $c(\alpha=0.01, S)$ for the Kolmogorov-Smirnov test statistic $a=\max \left[\mid F_{\mathrm{S}}(u)-\right.$ $F(u) \mid] . H_{\mathrm{G}}$ is rejected if, and only if,

$a \geq c(\alpha=0.01, S)$. 
BL Her: $\quad P_{1}=1.307465 \pm 0.000034\left(\chi^{2}=188.5\right)$
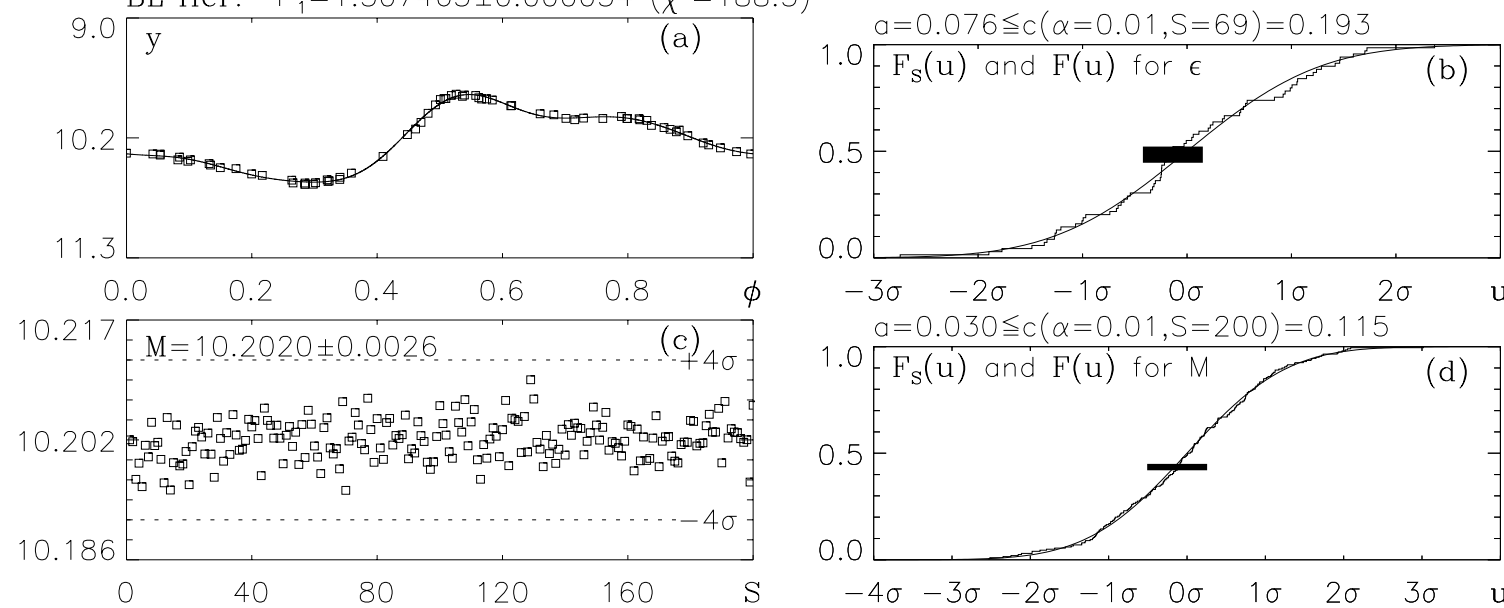

$a=0.030 \leqq c(\alpha=0.01, S=200)=0.115$
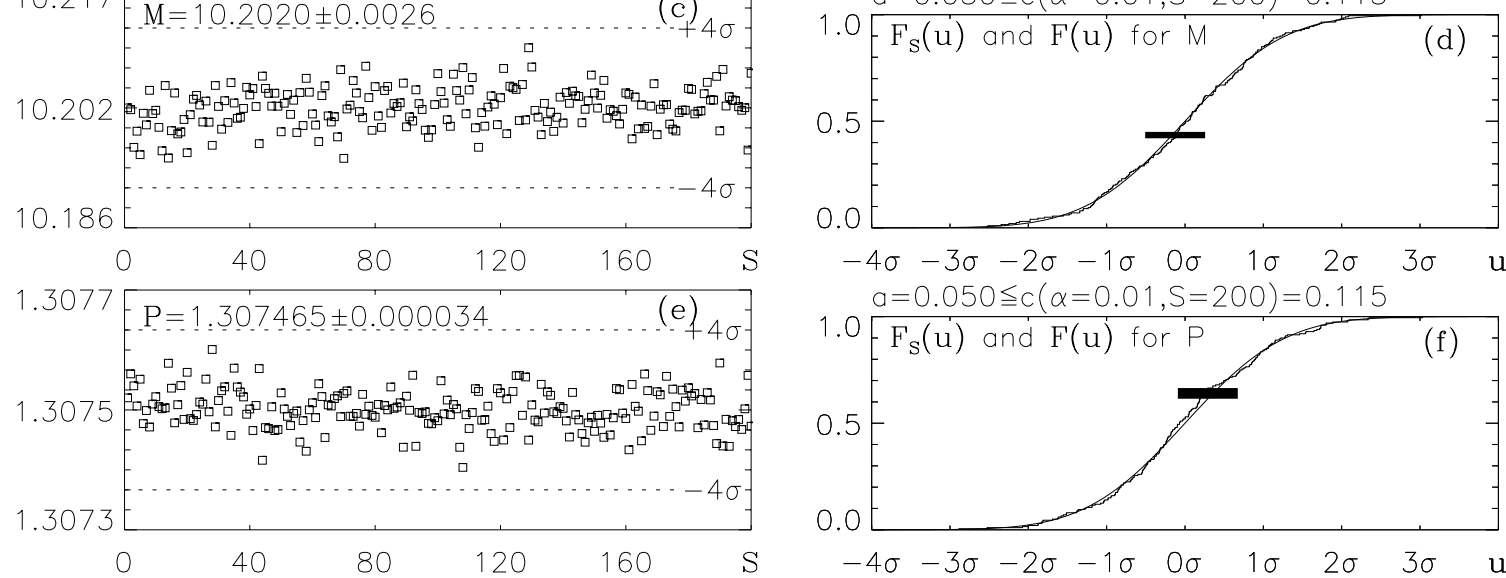

$a=0.050 \leqq c(\alpha=0.01, S=200)=0.115$

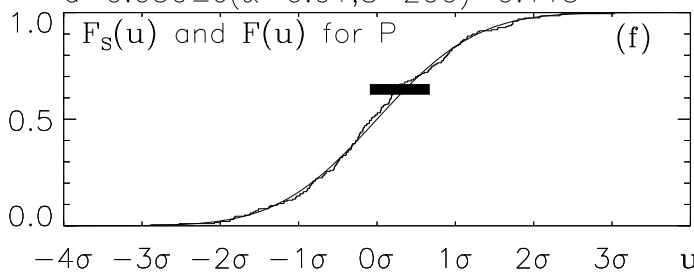

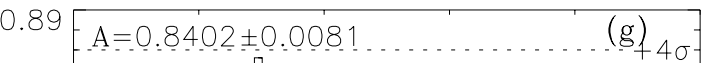
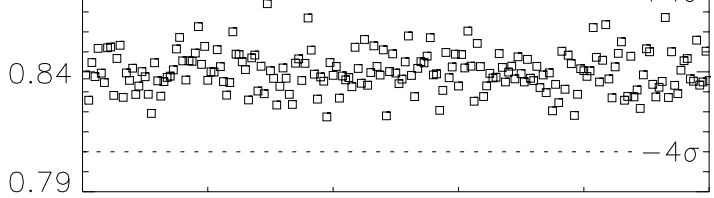

$\begin{array}{llllll}0 & 40 & 80 & 120 & 160\end{array}$
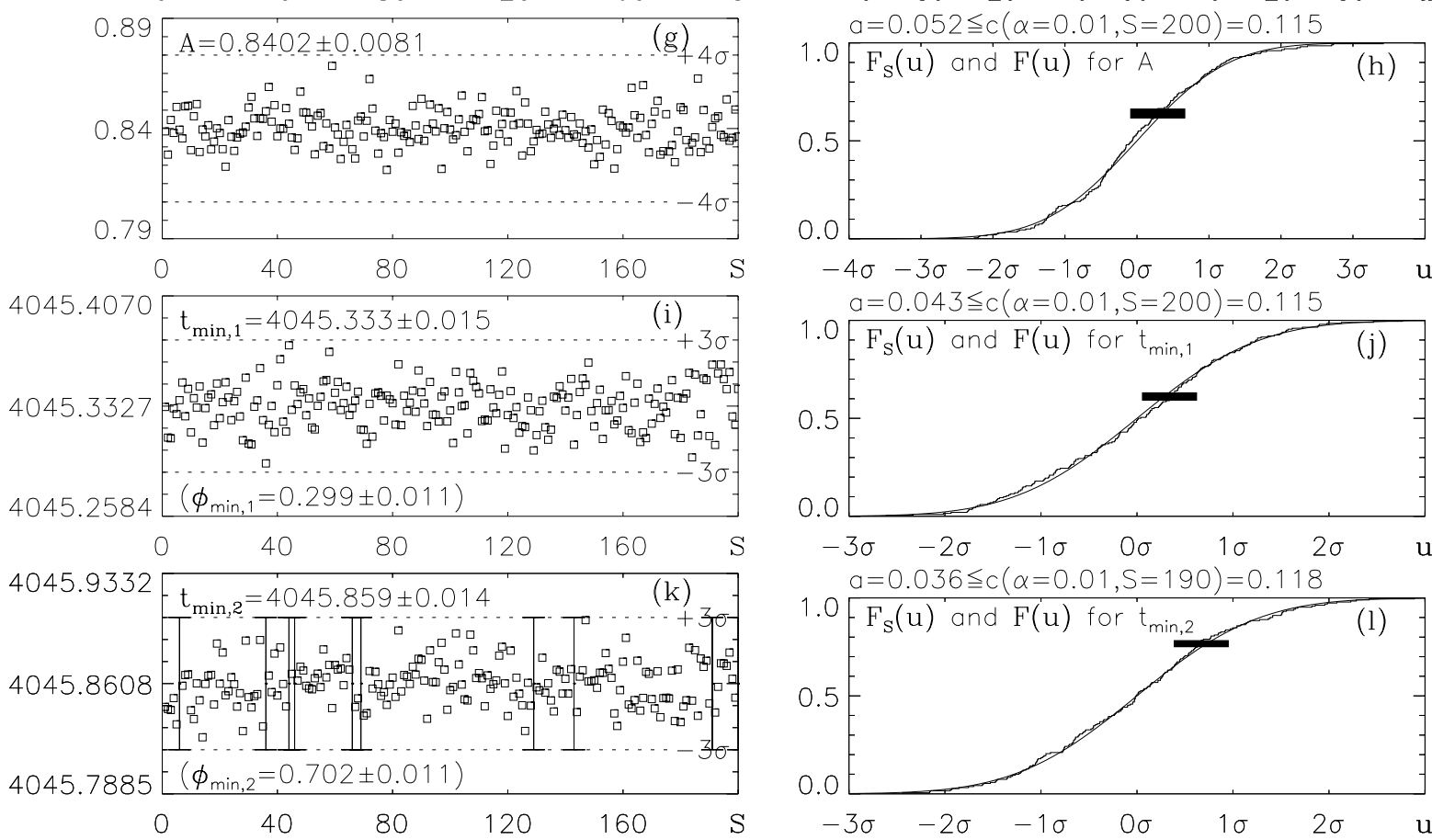

Fig. 6. The $S=200$ fifth order RSch bootstrap with $P_{1}=1.307465$ for the $V$ magnitudes of BL Her, otherwise as in Fig. 2 . Note: k) Only $S=190$ estimates are obtained for $t_{\min , 2}$

One might (correctly) argue that preserving the connection between $\epsilon_{i}$ and $w_{i}$ during the 2nd stage of the above bootstrap distorts the statistics in case of inhomogeneous data quality. In that case some low quality data with large $\epsilon_{i}$ and small $w_{i}$ will be randomly distributed among high quality data in each bootstrap sample. But this problem is eliminated by checking if the $\bar{\epsilon}$ distribution is Gaussian. If $H_{\mathrm{G}}$ is rejected for $\bar{\epsilon}$ with Eq. (14), i.e. the data quality is inhomogeneous, then the modelling is rejected (see $R_{\mathrm{I}}$ in Sect. 6.3). If, on the other hand, $H_{\mathrm{G}}$ is not rejected for $\bar{\epsilon}$, preserving the connection between $\epsilon_{i}$ and $w_{i}$ in our bootstrap is justified.

\section{Spurious periodicities}

The period with the smallest $\chi^{2}$ is not always "real", because "spurious" periods are introduced by the interplay 


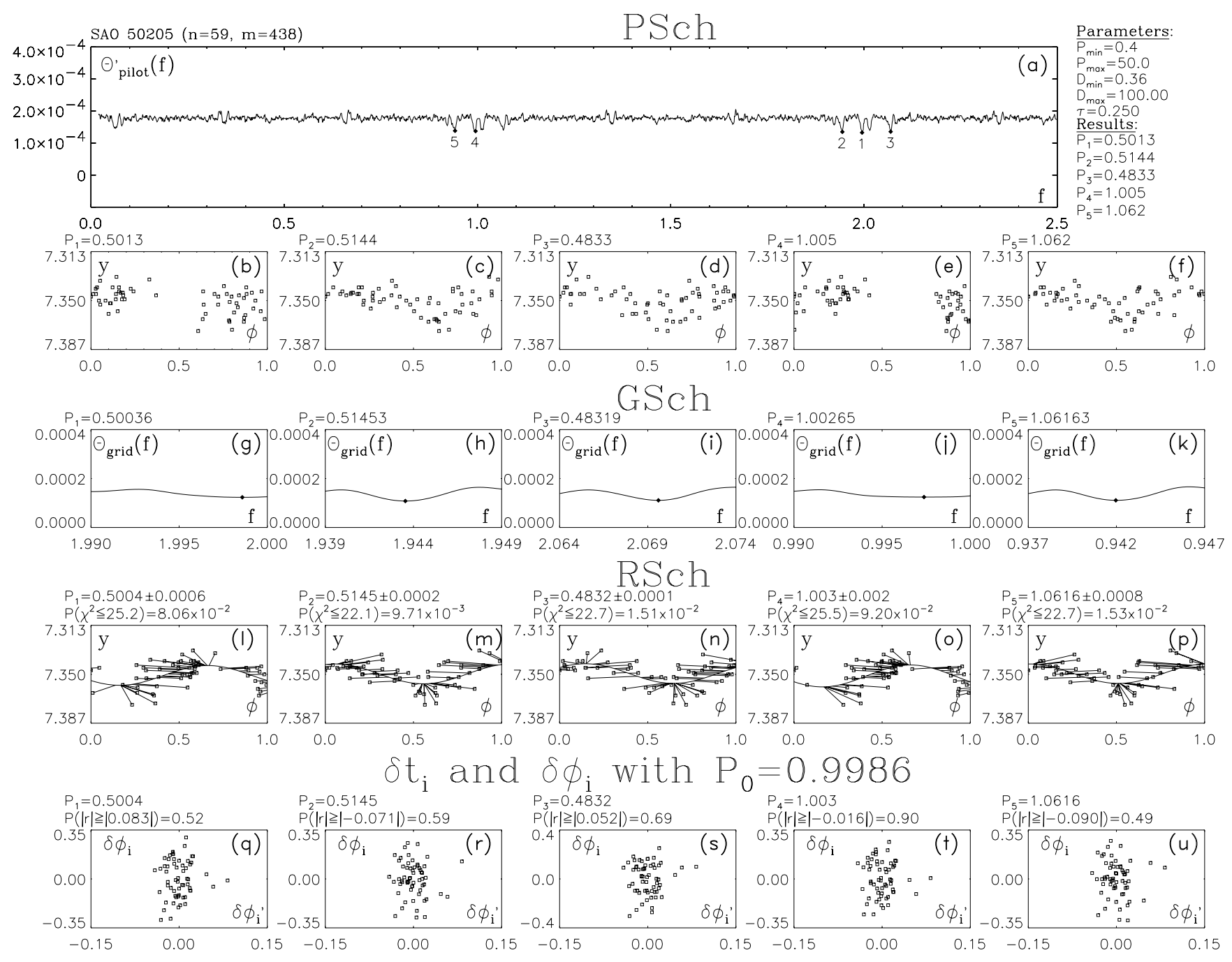

Fig. 7. The first order TSPA $(K=1)$ between $P_{\min }=0.4$ and $P_{\max }=50$ for the $V$ magnitudes of saO50205 during subsets $\mathrm{SET}=111,112,113$ and 114, otherwise as in Fig. 1

between $\bar{t}$ distribution and real periodicity. The phases of $\bar{t}$ folded with an arbitrary period are circular data. This $\bar{t}$ distribution can be studied with numerous methods for analysing circular data (e.g. Batshelet 1981; Jetsu \& Pelt 1996). We apply the Deeming (1975) window function

$\gamma_{n}(f)=n^{-1} \sum_{k=1}^{n} \mathrm{e}^{2 \pi i f t_{k}}$,

where the $n$ complex vectors $\left[\cos 2 \pi f t_{k}, i \sin 2 \pi f t_{k}\right]$ are more parallel for a significant window period $P_{0}=f^{-1}$. If $\bar{y}$ varies with $P_{1}$, then $P_{0}$ induces an important set of spurious periods

$P^{\prime}=P^{\prime}\left(P_{0}: k_{1}: k_{2}\right)=\left[P_{1}^{-1}+k_{1}\left(k_{2} P_{0}\right)^{-1}\right]^{-1}$,

where $k_{1}= \pm 1, \pm 2, \ldots$ and $k_{2}=1,2, \ldots$ This relation (Tanner 1948) states that $t_{i}$ separated by $k_{2} P_{0}$ have an integer phase difference $k_{1}$ with the periods $P_{1}$ and $P^{\prime}$. These $P^{\prime}\left(P_{0}: k_{1}: k_{2}\right)$ do not significantly alter the $\bar{y}$ sequence in $\bar{\phi}$. However, $P^{\prime}<0$ produce a "mirror image" by reversing the $\bar{\phi}$ sequence.
But Eq. (16) cannot separate $P_{1}$ from $P^{\prime}\left(P_{0}: k_{1}: k_{2}\right)$. The following modification of another method by Tanner (1948) may reveal, if a window $P_{0}$ induces a spurious period $P_{1}$ : (1) Derive $\delta \phi_{i}^{\prime}=\operatorname{FRAC}\left[\left(t_{i}-t_{1}\right) P_{0}^{-1}\right]$. Transform $\delta \phi_{i}^{\prime}>0.5$ to $\delta \phi_{i}^{\prime}=\delta \phi_{i}^{\prime}-1$. (2) Model the $\bar{y}$ with $P_{1}$, and measure the "phase residuals" $\left(\delta \phi_{i}\right)$, i.e. the minimum $\phi$ distance for each $y_{i}$ from the model $g(\phi)$. These $\delta \phi_{i}$ are unique, when the distance between data $\left(\phi_{i}, y_{i}\right)$ and model $(\phi, g(\phi))$ is defined as $d^{2}=d_{1}^{2}+d_{2}^{2}$, where $d_{1}=\left(\phi-\phi_{i}\right)$ and $d_{2}=\left|y_{i}-g(\phi)\right|\{\max [g(\phi)]-\min [g(\phi)]\}^{-1}$. Transform $d_{1}>0.5$ to $d_{1}=d_{1}-1$. The $d_{1}$ and $d_{2}$ are comparable, because $d_{2}$ is independent of the $y_{i}$ units. The $d$ minimum determines the point $(\phi, g(\phi))$ closest to $\left(\phi_{i}, y_{i}\right)$ uniquely, which yields the phase residuals $\delta \phi_{i}=\phi-\phi_{i}$. Transform $\delta \phi_{i}>0.5$ to $\delta \phi_{i}=\delta \phi_{i}-1$. (3) The linear correlation coefficient $\left(\left|r_{0}\right|\right)$ between $\delta \bar{\phi}^{\prime}$ and $\delta \bar{\phi}$ is higher for "spurious" than "real" periods. Note that we subtract the means from $\delta \bar{\phi}^{\prime}$ and $\delta \bar{\phi}$ (Figs. $1 \mathrm{q}-\mathrm{u}, 3 \mathrm{q}-\mathrm{u}, 5 \mathrm{q}-\mathrm{u}$ and $7 \mathrm{q}-\mathrm{u}$ ), since 

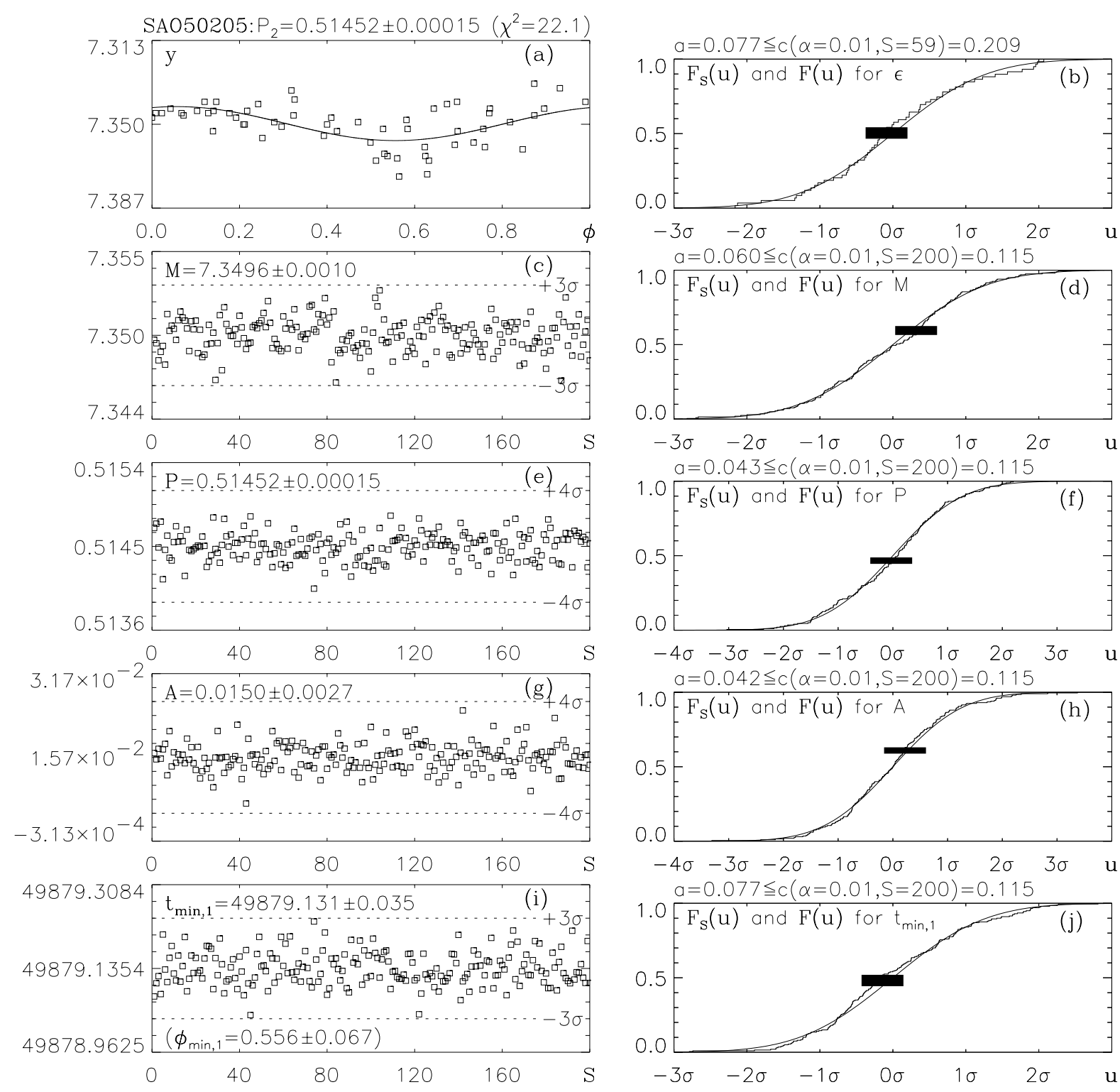

Fig. 8. The $S=200$ first order RSch bootstrap with $P_{2}=0.51452$ for the $V$ magnitudes of sAO50205, otherwise as in Fig. 2

this does not influence $\left|r_{0}\right|$. Our "correlation hypothesis" is

$H_{\mathrm{R}}$ : The $\delta \bar{\phi}^{\prime}$ and $\delta \bar{\phi}$ are uncorrelated random samples.

Under $H_{\mathrm{R}}$, the value of $P\left(r_{0}\right)=P\left(|r| \geq\left|r_{0}\right|\right)$ is the probability (i.e. the critical level) that $|r|$ reaches $\left|r_{0}\right|$, or an even larger, value.

\section{Application examples}

In this section we present detailed TSPA analysis examples for real data. All time units are days. Sections 6.1 and 6.2 describe the second order TSPA $(K=2)$ analysis for the normalized magnitudes of V 1794 Cyg during sub- sets $\mathrm{SET}=42$ and 114 from Jetsu ${ }^{1}$ et al. (1999b, Paper II). The next Sect. 6.3 clarifies our methodology in Paper I, where the second order TSPA analysis is performed for all subsets of normalized magnitudes of V 1794 Cyg. In Sect. 6.4 we apply the fifth order TSPA $(K=5)$ to the $V$ light curve of the cepheid variable BL Her (Moffett \& Barnes 1984). One sample of the $V$ magnitudes of the nonvariable primary comparison star SAO50205 from Paper II are analysed with the first order TSPA $(K=1)$ in Sect. 6.5.

\footnotetext{
1 The data in Paper II are available in electronic form: see the editorial in A\&A 1992, Vol. 266, No. 2, page E1.
} 


\subsection{Normalized UBVR magnitudes of $S E T=42$}

The magnitudes of V 1794 Cyg within each passband $(\bar{y})$ have been normalized with

$y_{\text {norm }}\left(t_{i}\right)=\left(y\left(t_{i}\right)-m_{y}\right)\left(H s_{y}\right)^{-1}$,

where $m_{y}$ and $s_{y}$ are the mean and standard deviation of $\bar{y}$, and $H$ is an arbitrary scaling factor. The $\bar{y}_{\text {norm }}$ standard deviation $s_{y, \text { norm }}=H^{-1}$ and $\bar{\sigma} s_{y}^{-1}=\bar{\sigma}_{\text {norm }} s_{\text {y,norm }}^{-1}$ give $\bar{\sigma}_{\text {norm }}=\left[H s_{y}\right]^{-1} \bar{\sigma}$. The accuracy of each individual photometric observation is usually unknown, like in Paper II that contains published and new photometry from several observatories. The $\bar{y}_{\text {norm }}$ and $\bar{\sigma}_{\text {norm }}$ were determined with $\sigma=0{ }^{\mathrm{m}} 015$ in $B V R, \sigma=0{ }^{\mathrm{m}} 030$ in $U$, and $H=4$ in Eq. (17). These normalized magnitudes are hereafter denoted by $\bar{y}$ and $\bar{\sigma}$, i.e. the "norm" subscripts are omitted here, as well as in Sects. 6.2 and 6.3.

In $\mathrm{SET}=42$, the deepest $\Theta_{\text {pilot }}^{\prime}(f)$ (Eq. 6) minimum between $P_{\min }=0.5$ and $P_{\max }=10$ is at $P_{1}=3.33$ (Fig. 1a: $n=149)$. The best window period $P_{0}=0.9997$ detected with $\gamma_{\mathrm{n}}$ (Eq. 15) connects $P_{1}$ to $P_{2}=0.77 \approx P^{\prime}\left(P_{0}: 1\right.$ : $1)=0.77$ and $P_{3}=1.42 \approx\left|P^{\prime}\left(P_{0}:-1: 1\right)\right|=|-1.43|$, while $P_{4} \approx 2 P_{2}$ and $P_{5} \approx 2 P_{1}$. The $y_{\mathrm{i}}$ curves resemble sinusoids with $P_{1}, P_{2}$ and $P_{3}\left(k_{1}=-1\right.$ : "mirror image"), and those with $P_{4}$ and $P_{5}$ double sinusoids (Figs. 1b-f). The $\Theta_{\text {grid }}(f)$ minima provide more accurate $P_{1}, \ldots, P_{5}$ (Figs. $1 \mathrm{~g}-\mathrm{k}$ ), and the RSch trial values $\bar{\beta}_{0}$. The RSch bootstrap with $S=200$ gives the final $P_{1}, \ldots, P_{5}$. Of these, $P_{1}=3.361 \pm 0.004$ has the smallest $\chi_{0}^{2}=165.9$, that for $P_{5} \approx 2 P_{1}$ being comparable (Figs. 1l-p). The critical levels for rejecting $H_{0}$ with $m=55$ are not significant, not even for $P_{1}$ (Fig. 11: $\left.P\left(\chi_{0}^{2}\right)=1.00\right)$. Three alternatives could explain this: (1) The errors $\bar{\sigma}$ are not correct, and $\chi_{0}^{2}$ is unreliable. (2) The errors are correct, but the model is not, e.g. the order $K=2$ may be too low, or perhaps the light curve evolves during $\Delta T$. (3) A few erroneous observations can contaminate the $\chi_{0}^{2}$ determined by four normalized magnitudes at each $t_{i}$ (i.e. one in each $U B V R$ passband). Hence we can not use $P\left(\chi_{0}^{2}\right)<0.01$ to reject $H_{0}$ for these data. Paper I ( $A_{V}$ changes in Sect. 3.2.) gives a counterexample, where TSPA modelling can apply this criterion. The lines connecting each $\left(\phi_{i}, y_{i}\right)$ to the closest point of the model $(\phi, g(\phi))$ determine the phase residuals $\delta \phi_{i}$ (Figs. 11-p). The linear correlation coefficient $\left|r_{0}\right|$ between $\delta \bar{\phi}^{\prime}$ and $\delta \bar{\phi}$ is smallest for $P_{1}$, but of the same order as that for $P_{5}$ (Figs. 1q and u). The highly improbable $\left|r_{0}\right|$ for $P_{2}$, $P_{3}$ and $P_{4}$ reveal that these periods are indeed spurious. In conclusion, the best period is $P_{1}$ with the smallest $\chi_{0}^{2}$ and lowest $\left|r_{0}\right|$. The double sinusoid with $P_{5}$ imitates the solution with $P_{1}$.

The $S=200$ RSch bootstrap not only provides the $P$ error estimate, but also those of $M$ (mean), $A$ (total amplitude), $t_{\min , 1}$ and $t_{\min , 2}$ (epochs of primary and secondary minima). Figure 2 summarizes the earlier $P_{1}$ bootstrap of Fig. 11. The $F_{\mathrm{S}}(u)$ (Eq. 12) for the $S=149$ (= $n$ ) residuals $\bar{\epsilon}$ does not cause $H_{\mathrm{G}}$ rejection with $\alpha=0.01$
(Fig. 2b, Eq. 14), i.e. this "empirical distribution" of $\bar{\epsilon}$ (Eq. 11) is Gaussian. The $M$ and $P$ distributions are also Gaussian (Figs. 2c-f). The model gives these theoretical $M$ and $P$ estimates directly, but those of $A$ and $t_{\min , 1}$ are measured from the model for each $\bar{y}^{*}$ to avoid the complicated theoretical solutions with $K \geq 2$. That these $A$ and $t_{\mathrm{min}, 1}$ distributions are also Gaussian, confirms the validity of our measurement approach (Figs. $2 \mathrm{~g}-$ j). Note that SET $=42$ has no secondary minimum $t_{\min , 2}$ with $P_{1}=3.361$, and that the primary minimum phase is $\phi_{\min , 1}=\operatorname{FRAC}\left[t_{\min , 1}-t_{1}\right]$ (Fig. 2i). In conclusion, the $\bar{\epsilon}$, $\bar{M}, \bar{P}, \bar{A}$ and $\bar{t}_{\mathrm{min}, 1}$ bootstrap statistics for $\mathrm{SET}=42$ are reliable.

The model parameter error estimates in this paper utilize $S=200$ bootstrap samples during the RSch. This ensures reliable statistics, because these estimates usually stabilize already at $S \lesssim 50$. For example, increasing the number of samples from $S=25$ to 200 in the RSch of Figs. 2c and e gave the following error estimates for the mean $\left(\sigma_{\mathrm{M}}\right)$ and the period $\left(\sigma_{\mathrm{P}}\right)$

\begin{tabular}{ccccccc}
\hline$S$ & 25 & 50 & 75 & 100 & 150 & 200 \\
\hline$\sigma_{\mathrm{M}}$ & 0.0058 & 0.0054 & 0.0050 & 0.0052 & 0.0051 & 0.0052 \\
$\sigma_{\mathrm{P}}$ & 0.0042 & 0.0041 & 0.0040 & 0.0040 & 0.0042 & 0.0042 \\
\hline
\end{tabular}

\subsection{Normalized UBV magnitudes of $S E T=114$}

The TSPA for SET $=42$ posed no serious problems (Sect. 6.1), but "pathological" cases are not rare for real data, and analysing the normalized $U B V$ magnitudes of $\mathrm{SET}=114$ serves as such a "good" counterexample. The "real" $P_{2}=3.33$ periodicity is not the best one detected by PSch (Fig. 3a), and TSPA reveals that $P_{1} \approx 0.77$ has the smallest $\chi_{0}^{2}$ (Figs. $31-\mathrm{p}$ ). The $P_{1}, \ldots, P_{5}$ and $P_{0}=0.9981$ connections are obvious (Eq. 16). All five periods reach extreme critical levels $\left(10^{-8} \lesssim P\left(\chi_{0}^{2}\right) \lesssim 10^{-6}\right)$, although the residuals appear extraordinary large. These $P\left(\chi_{0}^{2}\right)$ are extreme, because the data accuracy is equal or below the $U B V$ light curve amplitudes $A_{U}=0.020, A_{B}=0.014$ and $A_{V}=0.025$ during $\mathrm{SET}=114$ (Paper I, Table 1). But lowering the modelling order, e.g. $K=0 \equiv \bar{g}=$ constant, would confuse the time series analysis, it being crucial that the same model is used for all subsets to obtain $M, A, P, t_{\min , 1}$ and $t_{\min , 2}$. As expected, the $P_{2}=3.34 \pm 0.05$ periodicity during $\mathrm{SET}=114$ (Fig. $3 \mathrm{~m}$ ) is less accurate than that of $P_{1}=3.361 \pm 0.004$ during SET $=42$ with high $A_{V}=0.192$ (Paper I, Table 1). Nevertheless, the 3.34 signal detection in $\mathrm{SET}=114$ confirms that we are not modelling "noise". The low $U B V$ amplitudes in $\mathrm{SET}=114$ also explain the absence of correlations in Figs. $3 \mathrm{q}-\mathrm{u}$. The $S=200$ RSch bootstrap reveals other "unpleasant" results for $\mathrm{SET}=114$ (Fig. 4): The $P$ distribution is not Gaussian (Figs. 4e and $\mathrm{f})$, and a secondary minimum $\left(t_{\min , 2}\right)$ is present, but only in $S=163$ samples (Figs. $4 \mathrm{k}$ and l). The next 
Table 1. TSPA results between $P_{\min }=0.5$ and $P_{\max }=10$ for 93 subsets of normalized photometry of V $1794 \mathrm{Cyg}$

\begin{tabular}{lccccc}
\hline & $P \sim 3.33$ & $P^{\prime}\left(P_{0}: 1: 1\right) \sim 0.77$ & $-P^{\prime}\left(P_{0}:-2: 1\right) \sim 0.58$ & $-P^{\prime}\left(P_{0}:-1: 1\right) \sim 1.42$ & $2 P \sim 6.66$ \\
\hline Among detected $P_{1}, \ldots, P_{5}$ & $\frac{84}{93}(90 \%)$ & $\frac{83}{93}(89 \%)$ & $\frac{61}{93}(66 \%)$ & $\frac{77}{93}(83 \%)$ & $\frac{25}{93}(27 \%)$ \\
Smallest $\chi_{0}^{2}$ of $P_{1}, \ldots, P_{5}$ & $\frac{31}{84}(37 \%)$ & $\frac{22}{83}(27 \%)$ & $\frac{10}{61}(16 \%)$ & $\frac{8}{77}(10 \%)$ & $\frac{4}{25}(16 \%)$ \\
Smallest $\left|r_{0}\right|$ of $P_{1}, \ldots, P_{5}$ & $\frac{32}{84}(38 \%)$ & $\frac{12}{83}(14 \%)$ & $\frac{7}{61}(11 \%)$ & $\frac{13}{77}(17 \%)$ & $\frac{7}{25}(28 \%)$ \\
$P\left(\left|r_{0}\right|\right) \leq \alpha=0.01$ & $\frac{4}{84}(5 \%)$ & $\frac{11}{83}(13 \%)$ & $\frac{15}{61}(25 \%)$ & $\frac{16}{77}(21 \%)$ & $\frac{2}{25}(8 \%)$ \\
\hline
\end{tabular}

Sect. 6.3 clarifies how this type of apparently confusing results were interpreted in Paper I.

\subsection{All normalized subsets}

For $K=2$ and $S=200$ in Paper I, three rules were found sufficient for the TSPA modelling of any particular SET of normalized magnitudes of V 1794 Cyg:

- $R_{\mathrm{I}}$ : If $H_{\mathrm{G}}$ is rejected with $\alpha=0.01$ (Eq. 14) for any of the $\bar{\epsilon}, \bar{M}, \bar{P}, \bar{A}, \bar{t}_{\mathrm{min}, 1}$ or $\bar{t}_{\mathrm{min}, 2}$ distributions, then the $P, t_{\mathrm{min}, 1}$ and $t_{\mathrm{min}, 2}$ estimates are excluded from further analysis.

- $R_{\mathrm{II}}$ : The $P, t_{\min , 1}$ and $t_{\min , 2}$ of subsets with less than 10 observing nights (nts) are excluded from further analysis.

- $R_{\mathrm{III}}$ : A secondary minimum $t_{\mathrm{min}, 2}$ not present in $S \geq$ 190 (三95\%) bootstrap samples is excluded from further analysis. Note that $P$ or $t_{\min , 1}$ are not excluded.

For example, $H_{\mathrm{G}}$ is rejected for $P$ in $\mathrm{SET}=114$ (Fig. 4f), and $R_{\mathrm{I}}$ excludes the $P, t_{\mathrm{min}, 1}$ and $t_{\mathrm{min}, 2}$ estimates. $R_{\text {III }}$ alone would exclude $t_{\min , 2}$ with $S=163$ (Figs. $4 \mathrm{k}$ and $\mathrm{l})$. The mean $\left(m_{y}\right)$ and the standard deviation $\left(s_{y}\right)$ of the data in subsets with nts $\geq 7$ (i.e. all normalized subsets) are connected to the mean $(M)$ and the total amplitude $(A)$ of the TSPA model. The linear approximations $M \approx m_{y}$ and $A / s_{y} \approx 2.7$ are very accurate. Note that the criteria $R_{\mathrm{I}}, R_{\mathrm{II}}$ and $R_{\mathrm{III}}$ are therefore not used to reject the $M$ or $A$ estimates. Furthermore, $m_{y}$ and $s_{y}$ are independent of $P$, but $t_{\min , 1}$ and $t_{\min , 2}$ are not. Thus the TSPA (or any other) $M$ and $A$ modelling could be substituted with the above $m_{y}$ and $s_{y}$ relations in subsets with an adequate phase coverage. These $m_{y}$ and $s_{y}$ can be used for the same purposes (e.g. Donahue et al. $1997)$ that we use $M$ and $A$ in Paper I. Our $R_{\mathrm{II}}$ states the experimental result that the $R_{\mathrm{I}}$ rejections occur mostly with nts $<10$. This is understandable for $2 K+2=6$ free model parameters when one night covers only about $P_{\text {phot }} / 10$ for V 1794 Cyg. Table 3 in Paper I summarizes the TSPA of all normalized subsets of V $1794 \mathrm{Cyg}$, and the $R_{\mathrm{I}}, R_{\mathrm{II}}$ or $R_{\mathrm{III}}$ rejections.

Our Table 1 confirms that the $\sim 3.33$ periodicity is real, which would appear far from trivial, were there several subsets similar to $\mathrm{SET}=114$. We summarize the TSPA combined with the best window period $P_{0}$ determined separately for each SET of Paper I. The first line shows that $\sim 3.33$ is among the five best periods in 84/93 cases for
TSPA between $P_{\min }=0.5$ and $P_{\max }=10($ nine $\sim 3.33$ values in Table 3 of Paper I were detected with $P_{\text {min }}=2$ and $\left.P_{\max }=6\right)$. The spurious $\sim 0.77$ period is detected nearly as often $(83 / 93)$, but the detection rates for the other spurious periods are lower, especially for $\sim 6.66$. The next lines in Table 1 rate each period, if detected. The 2nd and 3rd lines confirm that $\sim 3.33$ is undoubtedly the best period with the $\chi_{0}^{2}$ (best model) and $\left|r_{0}\right|$ (not spurious) criteria. The 4 th line lists $H_{\mathrm{R}}$ rejections with $P\left(\left|r_{0}\right|\right) \leq \alpha=0.01$, i.e. when a high $\left|r_{0}\right|$ between $\delta \bar{\phi}^{\prime}$ and $\delta \bar{\phi}$ implies spurious periodicity. This occurs more frequently for periods other than $\sim 3.33$. Only $\sim 0.77, \sim 0.58$, $\sim 1.42$ or their multiples reach extreme critical levels (e.g. Figs. 1r-t: $\left.P\left(r_{0}\right) \lesssim 10^{-11}\right)$. In conclusion, our Table 1 illustrates the probability for obtaining a spurious (i.e. false) period for some arbitrary object.

\subsection{V light curve of $B L \mathrm{Her}$}

The above V 1794 Cyg light curve analysis was performed with a second order TSPA. Here we study the $V$ light curve of the cepheid variable BL Her from Moffett \& Barnes (1984). The complicated shape of this light curve required a higher order model. Several details of this shape are undoubtedly real, because the total amplitude of the brightness variations $(0.84)$ is nearly 100 times larger than the internal accuracy of these data. Our fifth order TSPA $(K=5)$ between $P_{\min }=0.5$ and $P_{\max }=10$ gave the best period of $P_{1}=1.30747 \pm 0.00003$ (Fig. 5), and confirmed the 1.307443 estimate in Moffett \& Barnes (1984). This periodicity certainly reached the best $\chi_{0}^{2}$ among all period candidates (Figs. 5l-p), but the 0.01 internal error in $V$ yields $\chi_{0}^{2}=188.5>n=69$. Considering that the external error of the transformation into the standard Johnson system was "nearly as good" as the internal error (Moffett \& Barnes 1984), the above $\chi_{0}^{2}$ should be divided by an unknown factor larger than two. Three of the other period candidates are nearly multiples of $P_{1}$, i.e. $P_{2}=2.00 P_{1}, P_{3}=3.07 P_{1}$ and $P_{4}=4.12 P_{1}$. The $P_{5}$ periodicity is also not induced by the $P_{0}=0.9996$ window period, but it may be connected to the $277^{\mathrm{d}}$ gap within the data and/or the $446^{\mathrm{d}}$ time span of the whole data. Since these spurious periodicities are not connected to $P_{0}$, the phase residual correlations are not significant (Figs. 5q-u). The RSch bootstrap with $P_{1}$ confirmed that the modelling statistics are reliable, because the $\bar{\epsilon}, \bar{M}, \bar{P}$, 
$\bar{A}, \bar{t}_{\mathrm{min}, 1}$ and $\bar{t}_{\mathrm{min}, 2}$ distributions are all Gaussian (Fig. 6). Note also that the secondary minimum $t_{\min , 2}$ is present in $S=190$ bootstrap samples (Figs. 6kl).

\subsection{V light curve of SAO 50205}

The nonvariable star SAO50205 (B8II) has been used as the primary comparison star in the differential photometry of V 1794 Cyg. The long-term constant brightness of this object was verified in Paper II, where it was also noted that no periodicity was detected in the short-term brightness. The $V$ magnitudes of sAO50205 measured with the Automatic Photoelectric Telecope (APT) during four subsets $\mathrm{SET}=111-114$ are studied here. If the selected model $\bar{g}$ for these $n=59$ values were their mean of 7.350 , the 0.012 external error of these APT data would give $\chi_{0}^{2}=34.5$ having $P\left(\chi_{0}^{2}\right)=610^{-3}$ (Eq. (10): $\nu=n-1$ and $m=1)$. In other words, a constant brightness model would suffice. But to illustrate a case of "pure noise", the analysis of these data with the first order TSPA $(K=1)$ between $P_{\min }=0.4$ and $P_{\max }=50$ is presented in Fig. 7 . The PSch periodogram $\Theta_{\text {pilot }}^{\prime}(f)$ is nearly featureless, except for the weak minima close to the frequencies of 1 and 2 (Fig. 7a). Such integer values usually indicate spurious periodicity. The five best period candidates were divided into two groups when inferring their origin.

The periods of $P_{1}$ and $P_{4}$ belong to the first group. They are window periods inducing long gaps in the phase distribution of the data (Figs. 7b and e). Both periodicities are connected to the best window period $P_{0}=0.9986$ and the whole $\Delta T=176.8$ time span of the data. The combinations are $P^{\prime}(\Delta T:-1: 1)=1.004 \approx P_{4}=1.003$ and $P_{4} / 2=0.5015 \approx P_{1}=0.5004$. The measurements follow a weak slope spanning about 0.5 in phase. The gaps in the phases of the data allow a reasonable fit of the $K=1$ model to these slopes.

The second group containing $P_{2}, P_{3}$ and $P_{5}$ is a more complicated case. These three periods are connected to $P^{\prime \prime}=16.8 \pm 0.4$, which would have been the best period, if the TSPA had been performed from $P_{\min }=2$ to $P_{\text {max }}=50$. The $\Theta_{\text {pilot }}^{\prime}(f)$ periodogram has one weaker minimum at $f^{\prime \prime}=1 / P^{\prime \prime} \approx 0.06$ (Fig. 7a). The model with $P^{\prime \prime}$ would reach $\chi_{0}^{2}=23.6$, which is comparable to $22.1 \leq \chi_{0}^{2} \leq 22.7$ with $P_{2}, P_{3}$ and $P_{5}$. Combining $P^{\prime \prime}$ to the window period $P_{0}=0.9986$ yields $P^{\prime}\left(P_{0}:-2: 1\right)=-0.5146 \approx$ $-P_{2}=0.5145, P^{\prime}\left(P_{0}: 2: 1\right)=0.4849 \approx P_{3}=0.4832$ and $P^{\prime}\left(P_{0}:-1: 1\right)=-1.0617 \approx-P_{5}=1.0616$. The low total amplitude of the corresponding models prevented us from utilizing the phase correlations in indentifying spurious periodicities (Figs. 7r, s and $\mathrm{u}$ ). This problem was solved by performing the same TSPA to the 19 other available subsets of $V$ photometry of SAO50205. These additional data revealed no signatures of $P^{\prime \prime}=16.8$. Hence $P^{\prime \prime}$ represents an artifact only present in SET $=111-114$, where it induces the "detection" of the $P_{2}, P_{3}$ and $P_{5}$ periodicities.
Since this TSPA tested $m=438$ independent frequencies, the "best" period of $P_{2}=0.5145 \pm 0.0002$ with $\chi_{0}^{2}=22.1$ does not reach the significance level of the constant brightness model with $\chi_{0}^{2}=34.5$, which was discussed before performing the TSPA analysis. Although the accuracy of the data $(0.012)$ was comparable to the total amplitude of the model $\left(0{ }^{\mathrm{m}} 015\right)$, there was no need to reject the Gaussian hypothesis $\left(H_{\mathrm{G}}\right)$ for any of the model parameter estimates in the RSch bootstrap with $P_{2}$ (Fig. 8). The above analysis of the $V$ magnitudes of SAO50205 illustrates the difficulties encountered in confirming the case of "pure noise" for real data with the $\chi_{0}^{2}$-criterion (Eq. 10). In conclusion, one must know the accuracy of the data and infer the contribution of the window period(s).

\section{Conclusions}

Time series analysis is a part of standard mathematical statistics. In time series analysis, we formulated the three stage period analysis (TSPA) capable of detecting any arbitrary periodic function in weighted data (Sect. 3). The free and "other" model parameter error estimates were then presented (Sect. 4), and the determination of data distribution in time (Sect. 5). In statistics, the idea that the TSPA represents $\chi^{2}$-tests performed for $m$ independent frequencies allowed significance estimates. The Kolmogorov-Smirnov test supplied further modelling information. The linear correlations between the phase residuals $\bar{\phi}^{\prime}$ and $\bar{\phi}$ were introduced for the spurious periodicity identification that is crucial, e.g. in multiperiodicity studies (Breger 1997). In general, all three stages of TSPA may sometimes be unnecessary, e.g. the analysis of some data might begin directly from the GSch.

One of our main goals was to provide the precise formulation of all methods applied in Paper I. Several examples of the TSPA analysis of real data were therefore presented in Sect. 6. Two subsets of the normalized photometry of V 1794 Cyg were studied in Sects. 6.1 and 6.2. The TSPA and complementary methods performed perfectly in the first case. The second case illustrated problematic results that are not at all rare for real data with any time series analysis method. Our methodology in analysing all 93 subsets of normalized photometry of V 1794 Cyg in Paper I was summarized in Sect. 6.3. A higher order model was illustrated in Sect. 6.4, where the fifth order TSPA was applied to the $V$ light curve of the cepheid variable BL Her. Even higher order models may be necessary, e.g. a TSPA with $K>5$ could be used to model the sharp primary and secondary minima interrupting the phases of constant brightness in eclipsing binaries, if the data phase coverage is adequate. The case of "pure noise" was studied in Sect. 6.5, where the first order TSPA was applied to the $V$ light curve of the nonvariable star SAO50205. 
We shall now compare our method to the popular Lomb-Scargle periodogram, i.e. the advantages and limitations of the TSPA with respect to the innumerable other available time series analysis methods are not reviewed in detail. Except for the $\Theta_{\text {grid }}$ scaling (Eq. 7), our GSch with $K=1$ and unity weights equals the Lomb-Scargle periodogram (Scargle 1982: “... fitting sine waves..."). In this case the RSch would give the same results, if combined to a high overfilling factor in the GSch, e.g. OFAC in Press \& Teukolsky (1988). But the Lomb-Scargle periodogram analysis fails when the correct model is $K \geq 2$ (e.g. with the $\chi^{2}$-criterion), while the TSPA with any $K$ utilizes this error information. The Lomb-Scargle periodogram has been compared to several other time series analysis methods, e.g. by Schwarzenberg-Czerny (1996) when introducing a method that substituted a Fourier series model of $K$ harmonics with an equivalent orthogonal polynomial series. It was also pointed out that the analysis of variance test statistic (ANOVA) combined to and applied in this orthogonal polynomial method is uniformly the most powerful test statistic. Schwarzenberg-Czerny (1996) emphasized that unlike ANOVA, many frequently applied test statistics are normalized by dividing with the empirical variance of the data, which causes unreliable significance estimates. We conclude that the $\chi_{0}^{2}$ significance estimates of the TSPA model are reliable, if only if the accuracy of the data is precisely known. But even when the unknown accuracy of individual measurements prevents significance estimates, cases like the analysis of the 93 subsets of V 1794 Cyg normalized photometry confirm that our method detects real periods and identifies spurious ones (Table 1). Furthermore, the bootstrap model parameter error estimates are always reliable, because these estimates are based on the modelling residuals that reflect the accuracy of the data, be it known or unknown. In conclusion, the TSPA and complementary methods, being relatively easy to apply and interpret, are efficient in searching for periodicity in large weighted time series (e.g. Paper I).

Acknowledgements. The work was partly supported by the EC Human Capital and Mobility (Networks) project "Late type stars: activity, magnetism, turbulence" No. ERBCHRXCT940483. We wish to thank the (anonymous) referee for valuable comments on the manuscript.

\section{References}

Batschelet E., 1981, Circular Statistics in Biology. Academic Press, London

Bossi M., La Franceschina L., 1995, A\&AS 113, 387

Breger M., Handler G., Garrido R., et al., 1997, A\&A 324, 566

Buccheri R., De Jager O.C., 1989, in Timing Neutron Stars, NATO ASI Series C, Vol. 262., In: Ögelman H., van den Heuvel E.P.J. (eds.). Kluwer Academic Publishers, Dordrecht, p. 95

Cuypers J., 1986, A\&A 167, 282

Deeming T.J., 1975, Ap\&SS 36, 137

Donahue R.A., Dobson A.K., Baliunas S.L., 1997, Solar Phys. 171, 211

Efron B., Tibshirani R., 1986, Stati. Sci. 1, 54

Evans D.S., 1961, Royal Obs. Bull. 30, E93

Foster G., 1996, AJ 111, 555

Jetsu L., Pelt J., 1996, A\&AS 118, 587

Jetsu L., Pelt J., Tuominen I., 1999a, A\&A (in press) (Paper I)

Jetsu L., Tuominen I., Bopp B.W., et al., 1999b, A\&AS, (in press) (Paper II)

Lomb N.R., 1976, Ap\&SS 39, 447

Martinez P., Koen C., 1994, MNRAS 267, 1039

Moffett T.J., Barnes T.G., 1984, ApJS 55, 389

Pelt J., 1980, Frequency Analysis of Astronomical Time Series, Valgus Publ. Tallinn, monograph, in Russian, p. 134

Pelt J., 1983, ESA SP-201, 37

Pelt J., 1992, Irregularly Spaced Data Analysis, User Manual, a program package, University of Helsinki, p. 267

Pelt J., 1993, in 5th ESO/ST-ECT Data Analysis Workshop, ESO Conference and Workshop Proceedings No. 47, in: Grosbøl P.J., Ruijsscher R.C.E. (eds.), p. 179

Pelt J., 1997, in Applications of Time Series Analysis in Astronomy and Meteorology, in: Subba Rao T., Priestley M.B., Lessi O. (eds.). Chapman \& Hall, p. 245

Press W.H., Teukolsky S.A., 1988, Computers in Physics, Nov./Dec. 1988 , p. 77

Press W.H., Flannery B.P., Teukolsky S.A., Vetterling W.T., 1988, Numerical Recipes. C. Cambridge University Press, New York

Scargle J.D., 1982, ApJ 263, 835

Schwarzenberg-Czerny A., 1996, ApJ 460, L107

Tanner R.W., 1948, Commun. from the David Dunlap Obs. University of Toronto, 16, p. 177

Taylor J., Hamilton S., 1972, Ap\&SS 17, 357

Vaniček P., 1969, Ap\&SS 4, 387

Vaniček P., 1971, Ap\&SS 12, 10

Wilcox J.Z., Wilcox T.J., 1995, A\&AS 112, 395 